\title{
1 Virtual drug repurposing study against SARS-CoV-2 TMPRSS2 target
}

\section{Serdar Durdagi}

ORCID ID: 0000-0002-0426-0905

Computational Biology and Molecular Simulations Laboratory, Department of Biophysics, School of Medicine, Bahcesehir University, Istanbul, Turkey

\section{Abstract}

Currently, the world suffers from a new coronavirus SARS-CoV-2 that causes COVID-19. Therefore, there is a need for the urgent development of novel drugs and vaccines for COVID19. Since it can take years to develop new drugs against this disease, here we used a hybrid combined molecular modeling approach in virtual drug screening repurposing study to identify new compounds against this disease. One of the important SARS-CoV-2 targets namely type 2 transmembrane serine protease (TMPRSS2) was screened with NPC's NIH small molecule library which includes approved drugs by FDA and compounds in clinical investigation. We used 6654 small molecules in molecular docking and top-50 docking scored compounds were initially used in short (10-ns) molecular dynamics (MD) simulations. Based on average MM/GBSA binding free energy results, long (100-ns) MD simulations were employed for the identified hits. Both binding energy results as well as crucial residues in ligand binding were also compared with a positive control TMPRSS2 inhibitor, Camostat mesylate. Based on these numerical calculations we proposed a compound (benzquercin) as strong TMPRSS2 inhibitor. If these results can be validated by in vitro and in vivo studies, benzquercin can be considered to be used as inhibitor of TMPRSS2 at the clinical studies.

Key Words: Drug repurposing, TMPRSS2, COVID19, SARS-CoV-2

\footnotetext{
*E-mail: serdar.durdagi@med.bau.edu.tr; durdagilab.com
} 


\section{Introduction}

Coronaviruses (CoVs) belong to a single-stranded RNA (positive-sense) virus family which is encapsulated by a membrane envelope. (Durdagi et al., 2020) There are four common types of coronavirus (alpha, beta, gamma and, delta) and cause mild upper respiratory tract diseases. (McKee et al., 2020) Human beta-coronaviruses are highly pathogenic. (Zhang et al., 2020) The SARS (Severe Acute Respiratory Syndrome)-CoV was appeared in Guangdong in China and infected 8096 people worldwide in 2002-2003. The fatality rate was around $10 \%$ (i.e., 774 deaths). In 2012, the MERS (Middle East Respiratory Syndrome)-CoV infected about 2500 people and the fatality rate was 36\%. (Blanco-Melo et al., 2020) Currently, the world suffers from a novel coronavirus SARS-CoV-2 that causes 2019 coronavirus disease (COVID19). Like SARS and MERS-CoVs, SARS-CoV-2 mainly affects the lower respiratory tract. (BlancoMelo et al., 2020) It is characterized by a number of symptoms such as fever, cough, diarrhea, and general weakness. (Blanco-Melo et al., 2020) In more serious cases, it causes acute respiratory distress syndrome and lung damage leading to inflammation and pneumonia. On the $11^{\text {th }}$ of March 2020, the WHO declared the COVID-19 as a pandemic. (Hoffmann et al., 2020) It affected the lives of hundreds of millions of people as a result of compulsory isolation and quarantines in the world. (Sanders et al., 2020) SARS-CoV-2 targets host cells via Spike protein that binds to the angiotensin-converting enzyme 2 (ACE2) receptor. The virus then uses host cell receptors (i.e., type 2 transmembrane serine protease (TMPRSS2)) and endosomes to enter the cells. TMPRSS2 facilitates cell entry of SARS-CoV-2 through the Spike protein. After entering the host cell, viral proteins are synthesized that encode for the replicase-transcriptase complex. (Mousavizadeh and Ghasemi 2020) Viral RNA was then synthesized by RNA-dependent RNA polymerase. (Durdagi et al., 2020) The genome of SARSCoV-2 encodes for different structural and non-structural proteins. (Sanders et al., 2020) The 
important ones for drug development studies are Main Protease, and RNA-dependent RNA polymerase. (Cao et al., 2020) Currently these proteins are mainly targeted for drug screening and drug repurposing studies.

It is noteworthy that the method known as drug repurposing has become less costly in terms of both time and resources. In our laboratory, the use of molecules in different indications has been investigated with the studies we have done in this field in recent years. (Durdagi et al., 2018; Is et al.,2018; Tutumlu et al., 2020) Since the most of the preclinic and clinical studies including pharmacokinetic and toxicological studies of approved or compounds in clinical investigation phases have been already tested, they require less time to make them suitable for new indications. The toxicity and ADME (absorption, distribution, metabolism and excretion) studies which need long time to complete are not required as the molecules considered in drug repositioning pass these stages already that should be applied before and have well-defined profiles. Therefore, their use in epidemics is more suitable than new molecules that have never been tested. Hence, in the current study we performed a virtual drug repurposing study. The small molecules from NCGC-NIH Chemical Genomics Center Pharmaceutical Collection (i.e., NPC library) were used in virtual screening studies at the active site of developed TMPRSS2 model target protein.

\section{Materials and Methods}

We downloaded and prepared 7922 small compounds from NPC library (https://tripod.nih.gov/npc/). Before the virtual screening, to remove non-specific compounds, we performed some filtration criteria. Such as molecular weights of compounds which are more than $1000 \mathrm{~g} / \mathrm{mol}$ and smaller than $100 \mathrm{~g} / \mathrm{mol}$ were removed from the library. The compounds that have more than 100 rotatable bonds or hydrogen bond acceptor and donor number that is higher than 10 were also removed. Thus, the total number of molecules 
were decreased to 6654 before the docking simulations. These compounds were prepared with LigPrep module (LigPrep, Schrodinger v.2017) of Maestro molecular modeling package. Since the crystal structure of the TMPRSS2 was not available, we performed homology modeling study. Swiss Model was used in homology modeling study (swissmodel.expasy.org).

The 5CE1 PBB coded serine protease hepsin was used as template structure. Sequence identity between template and target proteins was $35.2 \%$. Structural assessment studies on the developed model have been performed. There was no any residue at the unfavorable region at the Ramachandran's plot and protein reports showed no any steric clashes, bond length or bond angle deviations at the structure, thus it was suitable to use as target protein (Supplementary Materials, Figure S1). Before the docking, modeled target protein was prepared using Protein Preparation module of Maestro molecular modeling package. In the determination of protonation states of residues at the target protein, PROPKA was used. A restrained optimization protocol was employed with OPLS3e force field for the target protein model using $0.3 \AA$ A convergence criteria. Docking was implemented with Standard Precision (SP) protocol of Glide using default settings. For the top-50 docking poses all-atom molecular dynamics (MD) simulations were performed to mimic physiological conditions using Desmond. Orthorhombic box was used with explicit water models (i.e., TIP3P) that have $10 \AA$ thickness from the edges of the protein. $0.15 \mathrm{M} \mathrm{NaCl}$ added to the simulation box to neutralize the system. The particle mesh Ewald method was used for long range electrostatic interactions. 9.0 ̊̊ cut-off was used for both electrostatic and van der Waals interactions. The temperature $(310 \mathrm{~K})$ and pressure $(1.01325 \mathrm{bar})$ throughout the simulations were kept constant by Nose-Hoover thermostat (Evans and Holian, 1985) and Martyna-Tobias-Klein barostat (Martyna et al., 1994) The OPLS3e force field was used in MD simulations. 1000 trajectory frames for each system during the simulations were collected. Molecular Mechanics 
Generalized Born Surface Area (MM/GBSA) method was then employed for these trajectories and average MM/GBSA score for each studied compound was computed. The VSGB 2.0

100 solvation model in Prime module of Maestro was employed in MM/GBSA calculations.

\section{3. Results and Discussion}

102 Nowadays thanks to the effort of NIH Chemical Genomics Center, it is possible to obtain 103 electronic resources of collections of small molecules that have been registered. Combining

104 this with the power of recent advances of ligand- and target-driven based virtual screening methods, it has been made easier to consider drug repurposing for any disease especially for complex diseases. We used homology model target TMPRSS2 structure and screened the

107 prepared 6654 FDA approved drugs and compounds in clinical investigation phases from NPC 108 database using Glide/SP. Docking scores were between -8.445 and $3.438 \mathrm{kcal} / \mathrm{mol}$. (Figure S2)

109 Due to the limited flexibility of both the target protein and the screened ligands in docking,

110 the identification of the compounds based on only docking scores in docking simulations can

111 lead to false-positive results. (Tutumlu et al., 2020) Moreover, although molecular docking 112 simulations may provide an insight into protein/screened ligand interactions, it is always 113 important to understand how these interactions are sustained throughout the performing MD 114 simulations. Thus, we used top-50 docking scored compounds and employed initially short 115 (10-ns) all-atom MD simulations in physiological and body temperature conditions. Docking 116 scores of top 50 compounds were between -8.445 and $-6.962 \mathrm{kcal} / \mathrm{mol}$. (see Figure 1 and Table 117 S1 at the Supplementary Materials). An in-house script was used to prepare simulation boxes 118 and analyze MD simulations. Desmond was used in all MD simulations. Tables S1 represents 119 the average MM/GBSA scores using the recorded 1000 frames (strided by 10 during the 120 simulations in each system) of the top-50 ligands. In order to compare the docking scores and 121 average MM/GBSA scores of identified hit compounds through our virtual screening protocol, 
we also used same screening protocol for a well-known TMPRSS2 inhibitor, Camostat

123 mesylate. It is an approved small molecule compound for the treatment of pancreatitis in

124 Japan. It is found that Camostat mesylate prevents SARS-CoV-2 cell entry by inhibition of host serine protease TMPRSS2. Its docking score was $-5.444 \mathrm{kcal} / \mathrm{mol}$ at the binding pocket of the TMPRSS2. Its corresponding average MM/GBSA score was calculated as $-65.514 \mathrm{kcal} / \mathrm{mol}$.

127 Figure S3 at the Supplementary Materials show 2D ligand interactions diagram of Camostat 128 mesylate at the binding site of SARS-CoV-2 TMPRSS2 target. Crucial residues were found as Val280, Asp435, Ser436, GIn438, Gly439, Ser441, Ser460, and Val473. Based on average MM/GBSA scores of short MD simulations, we selected 3 hits that have better scores than -

$13170.0 \mathrm{kcal} / \mathrm{mol}$, which are Benzquercin, Difebarbamate and N-benzoyl-I-tyrosyl-paba. Long 132 (100-ns) MD simulations were performed for these compounds and MM/GBSA scores were 133 re-calculated. Results showed that Benzquercin, N-benzoyl-I-tyrosyl-paba and Difebarbamate 134 have average MM/GBSA scores of $-80.583,-56.162$, and $-66.567 \mathrm{kcal} / \mathrm{mol}$, respectively.

135 Results showed that especially Benzquercin (a flavonoid) has maintained the interactions with 136 the binding pocket residues. Although average MM/GBSA score of Difebarbamate is slightly 137 decreased at the long MD simulations compared to short simulation, it has still similar range 138 of MM/GBSA score with positive control compound Camostat. Figure 2 represents the 2D and $1393 \mathrm{D}$ ligand interactions diagram of Benzquercin at the binding site of TMPRSS2. The last frame 140 from 100-ns MD simulations was used. Crucial residues at the target were found as His296, 141 Glu299, Pro301, Leu302, Lys340, Lys342, Gly439, and Ser441 which share similar binding 142 pocket residues with positive control Camostat. Based on interaction fractions analysis Lys342 143 has the highest interaction fraction value with the hit compound throughout the 100-ns 144 simulations. The type of main interactions between the ligand and binding pocket residues 145 were hydrogen bonds through water bridges and hydrophobic interactions. 
146 Interestingly, benzquercin was also found as potent hit compounds in our previous virtual

147 screening study using same protocol against another important cell-entry target of SARS-CoV-

1482 Spike/ACE2. (Durdagi et al, 2020). Its average score at the Spike/ACE2 interface was found

149 as $-70.810 \mathrm{kcal} / \mathrm{mol}$. Hence, these studies showed that benzquercin may also act as dual

150 inhibitor. It has been recognized that the treatment of "one target/one molecule" approach

151 of complex diseases is not so effective. The usage of combined drugs is not also appreciated

152 due to toxicity and/or undesirable drug-drug interactions. The recent and promising approach

153 to these complex diseases including COVID-19 is instead to develop/identify unique

154 compounds that act on multi-targets simultaneously which these targets are crucial in the

155 studied disease. Thus, based on in silico results, benzquercin has this potential.

\section{4. Conclusions}

157 Molecular modeling studies, such as virtual screening, reduce the time required to set new

158 targets for known drugs and also provide the advantage of being cost-effective. (Durdagi et

159 al, 2018 and 2020; Shoichet et al, 2002). In this study, a virtual drug repurposing study was

160 performed to identify new compounds against TMPRSS2 which is an important target for the

161 entry of the SARS-CoV-2 to the host cell. Thus, NPC small molecule library was screened

162 initially by docking simulations. Based on docking scores, top-50 compounds were used in

163 short MD simulations and their average binding free energies were calculated by MM/GBSA

164 method. Selected hits were used in longer MD simulations and results showed that especially

165 benzquercin maintains its interactions with the crucial residues throughout the simulations. If

166 these results can be validated by experimental studies, benzquercin can be considered to be

167 used as inhibitor of TMPRSS2 at the clinical studies. 
171 This study was supported by Bahcesehir University, Bilimsel Araştırma Projeleri Birimi (BAP),

172 Project No: BAP.2020-01.01

173

174 Conflict of Interest

175 The author declares that there is no any conflict of interest.

176

177

References

178 Blanco-Melo D, Nilsson-Payant BE, Liu W-C, Uhl S, Hoagland D, Møller R, Jordan TX, Oishi K,

179 Panis M, Sachs D, Wang TT, Schwartz RE, Lim JK, Albrecht RA, tenOever BR. (2020) Imbalanced

180 Host Response to SARS-CoV-2 Drives Development of COVID-19, Cell, 181(5):1036-1045.

181

182 Cao Y, Li L, Feng Z, Wan S, Huang P, Sun X, Wen F, Huang X, Ning G, Wang W. (2020)

183 Comparative genetic analysis of the novel coronavirus (2019-nCoV/SARS-CoV-2) receptor

184 ACE2 in different populations. Cell Discovery 6:1-4.

185

186 Durdagi S, Aksoydan B, Erol I, Kantarcioglu I, Ergun Y, Bulut G, Acar M, Avsar T, Liapakis G,

187 Karageorgos V, Salmas RE, Sergi B, Alkhatib S, Turan G, Yigit BF, Cantasir K, Kurt B, Kilic T.

188 (2018) Integration of Multi-scale Molecular Modeling Approaches with Experiments for the in

189 silico Guided Design and Discovery of Novel hERG-Neutral Antihypertensive Oxazalone and

190 Imidazolone Derivatives and Analysis of Their Potential Restrictive Effects on Cell Proliferation.

191 European Journal of Medicinal Chemistry, 145:273-290

192 
193 Durdagi S, Aksoydan B, Dogan B, Sahin K, Shahraki A, Birgul-lyison N. (2020) Screening of

194 Clinically Approved and Investigation Drugs as Potential Inhibitors of SARS-CoV-2 Main

195 Protease and Spike Receptor-Binding Domain Bound with ACE2 COVID19 Target Proteins: A

196 Virtual Drug Repurposing Study. ChemRxiv, DOI: 10.26434/chemrxiv.12032712.v2

197

198 Evans DJ, Holian BL. The Nose-Hoover thermostat. (1985) The Journal of Chemical Physics.

199 83:4069-4074; DOI: 10.1063/1.449071

200

201 Hoffmann M, Kleine-Weber H, Krüger N, Herrler T, Erichsen S, Schiergens TS, Herrler G, Wu N202 H, Nitsche A, Müller MA, Drosten C, Pöhlmann S. (2020) SARS-CoV-2 Cell Entry Depends on 203 ACE2 and TMPRSS2 and Is Blocked by a Clinically Proven Protease Inhibitor. Cell, 181(2):271204280.

205

206 Is YS, Durdagi S, Aksoydan B, Yurtsever M. (2018) Proposing Novel MAO-B Hit Inhibitors Using 207 Multidimensional Molecular Modeling Approaches and Application of Binary QSAR Models for 208 Prediction of their Therapeutic Activity and Toxic Effects. ACS Chemical Neuroscience 9(7), $209 \quad 1768-1782$.

211 Martyna GJ, Tobias DJ, Klein ML. (1994) Constant pressure molecular dynamics algorithms.

212 The Journal of Chemical Physics. 101 (5):4177-4189.

214 McKee DL, Sternberg A, Stange U, Laufer S, Naujokat C. (2020) Candidate drugs against 215 SARS-CoV-2 and COVID-19 [published online ahead of print, 2020 Apr 29]. Pharmacological 216 Research, 157:104859. DOI:10.1016/j.phrs.2020.104859 
218 Mousavizadeh L, Ghasemi S. (2020) Genotype and phenotype of COVID-19: Their roles in

219 pathogenesis, Journal of Microbiology, Immunology and Infection, ISSN 1684-1182,

220 https://doi.org/10.1016/j.jmii.2020.03.022.

221

222 Sanders JM, Monogue ML, Jodlowski TZ, Cutrell JB. (2020) Pharmacologic Treatments for

223 Coronavirus Disease 2019 (COVID-19): A Review [published online ahead of print, 2020 Apr

224 13]. JAMA DOI: 10.1001/jama.2020.6019. DOI:10.1001/jama.2020.6019

225

226 Shoichet BK, McGovern, SL, Wei B, Irwin JJ (2002) Lead discovery using molecular docking.

227 Current Opinion in Chemical Biology 6: 439-446.

228

229 Tutumlu G, Dogan B, Avsar T, Orhan MD, Calis S, Durdagi S. (2020) Integrating Ligand and

230 Target-Driven Based Virtual Screening Approaches with in vitro human Cell Line Models to

231 Identify Novel Hit Compounds Against BCL-2. Frontiers in Chemistry, DOI:

$23210.3389 /$ fchem.2020.00167

233

234 Zhang R, Wang X, Ni L, Di Xiao, Ma Baitao, Niu S, Liu C, Reiter RJ (2020). COVID-19: Melatonin

235 as a potential adjuvant treatment. Life Sciences, 250:117583 DOI:

236 https://doi.org/10.1016/j.Ifs.2020.117583

237

238

239

240 


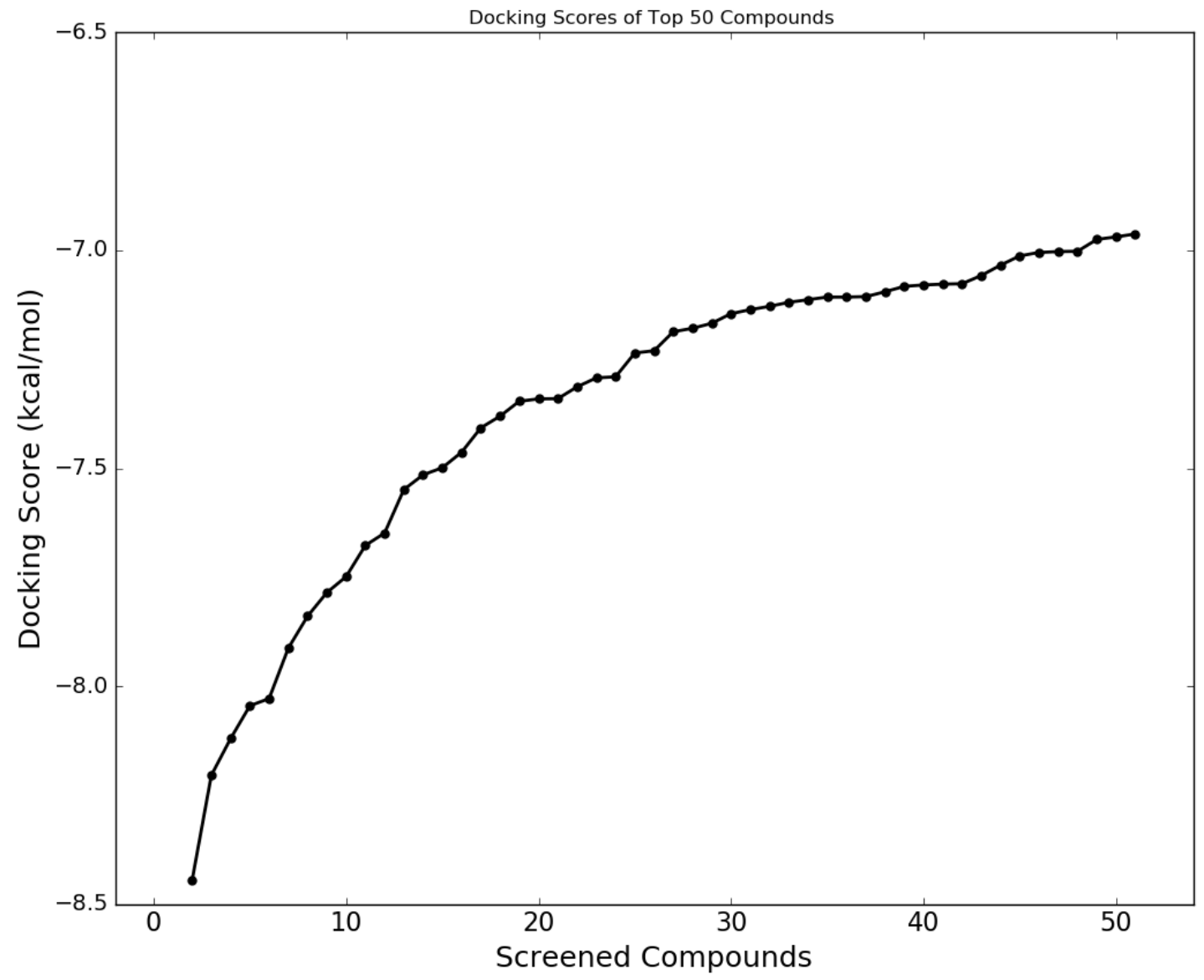

243 Figure 1. Docking scores of top-50 screened compounds from NPC database using Glide/SP.

244 

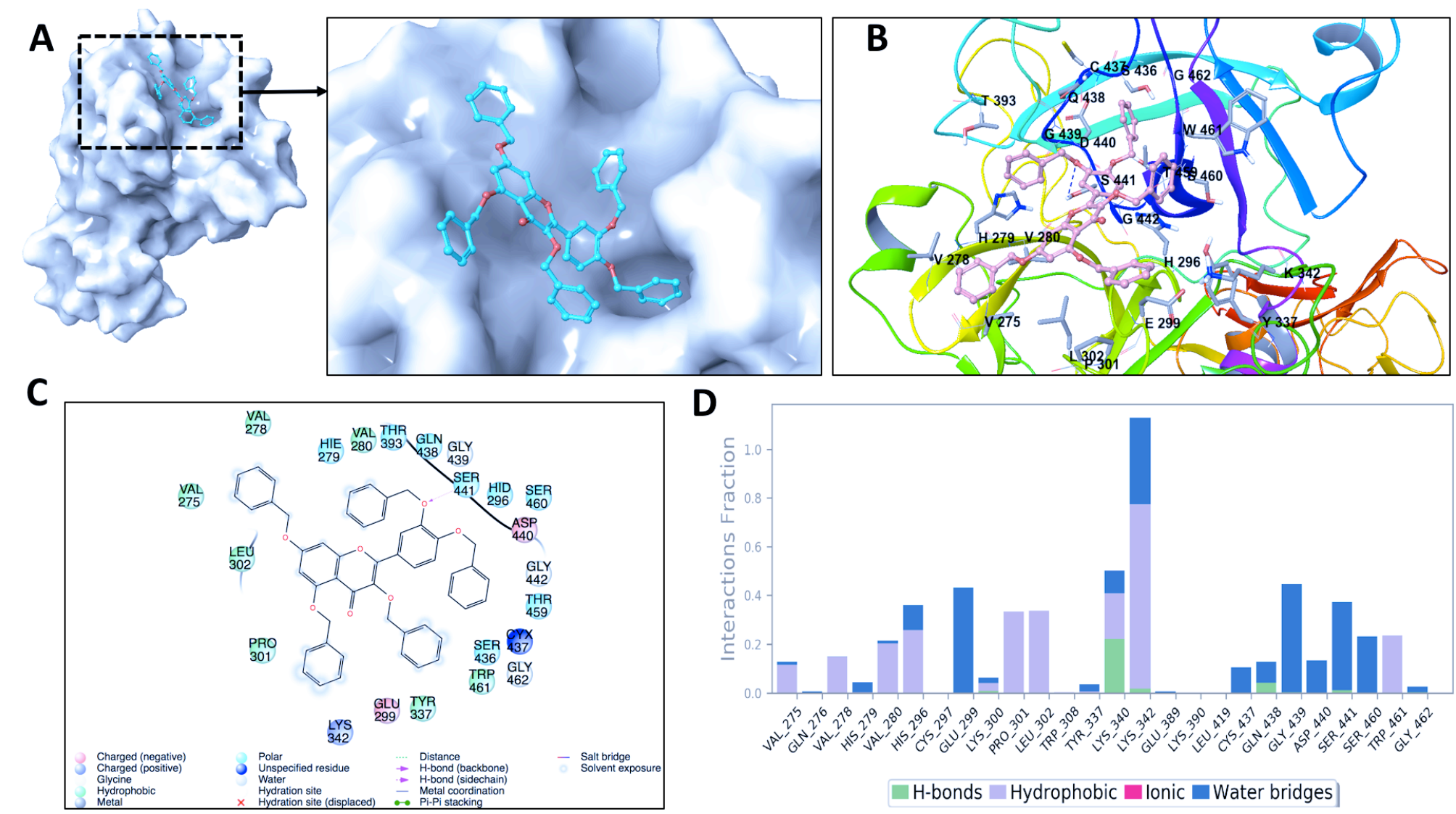

Figure 2. (A) Surface representation of benzquercin at the binding pocket of TMPRSS2. The last frame from 100-ns MD simulations was used.

250 (B) 3D ligand interactions diagram of benzquercin at the TMPRSS2 site. (C) Corresponding interactions were also depicted with 2D. (D) Interaction

251 fractions of binding pocket residues of TMPRSS2 with benzquercin throughout the MD simulations. Results show statistical results of collected 1000-trajectory frames throughout 100-ns MD simulations. 
Supplementary Materials 
A

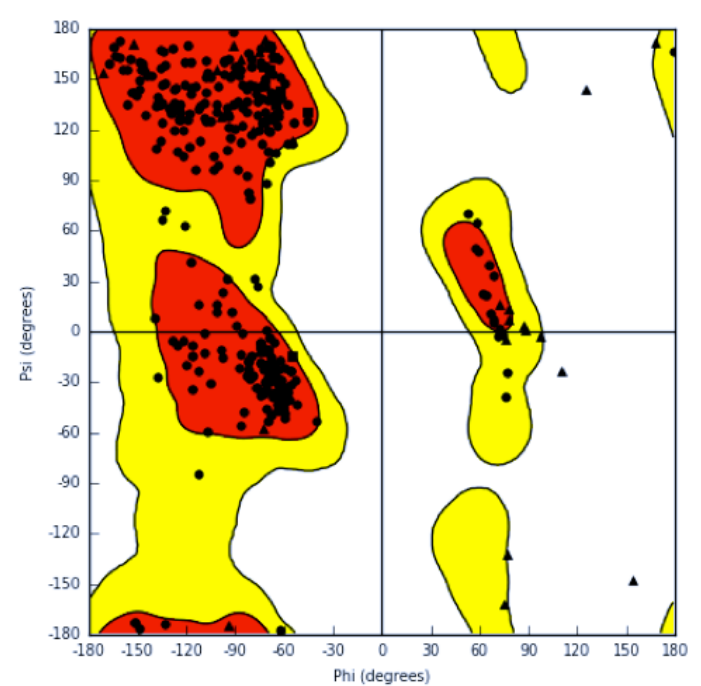

B

Local Quality Estimate
C
Comparison with Non-redundant Set of PDB Structures

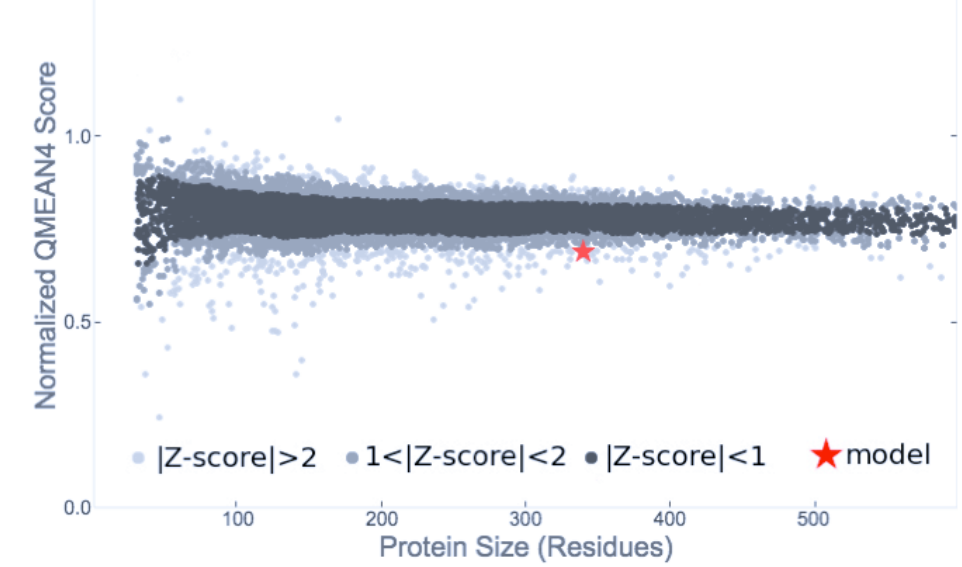

Figure S1. Structural assessment of developed homology model structure of TMPRSS2. (A) Ramachandran's plot. While filled spheres show each chiral residue at the target protein, filled triangles show Gly residues. (B) Local quality estimate of the derived model. (C) Normalized QMEAN4 score and its comparison with similar size of a set of PDB structures. 


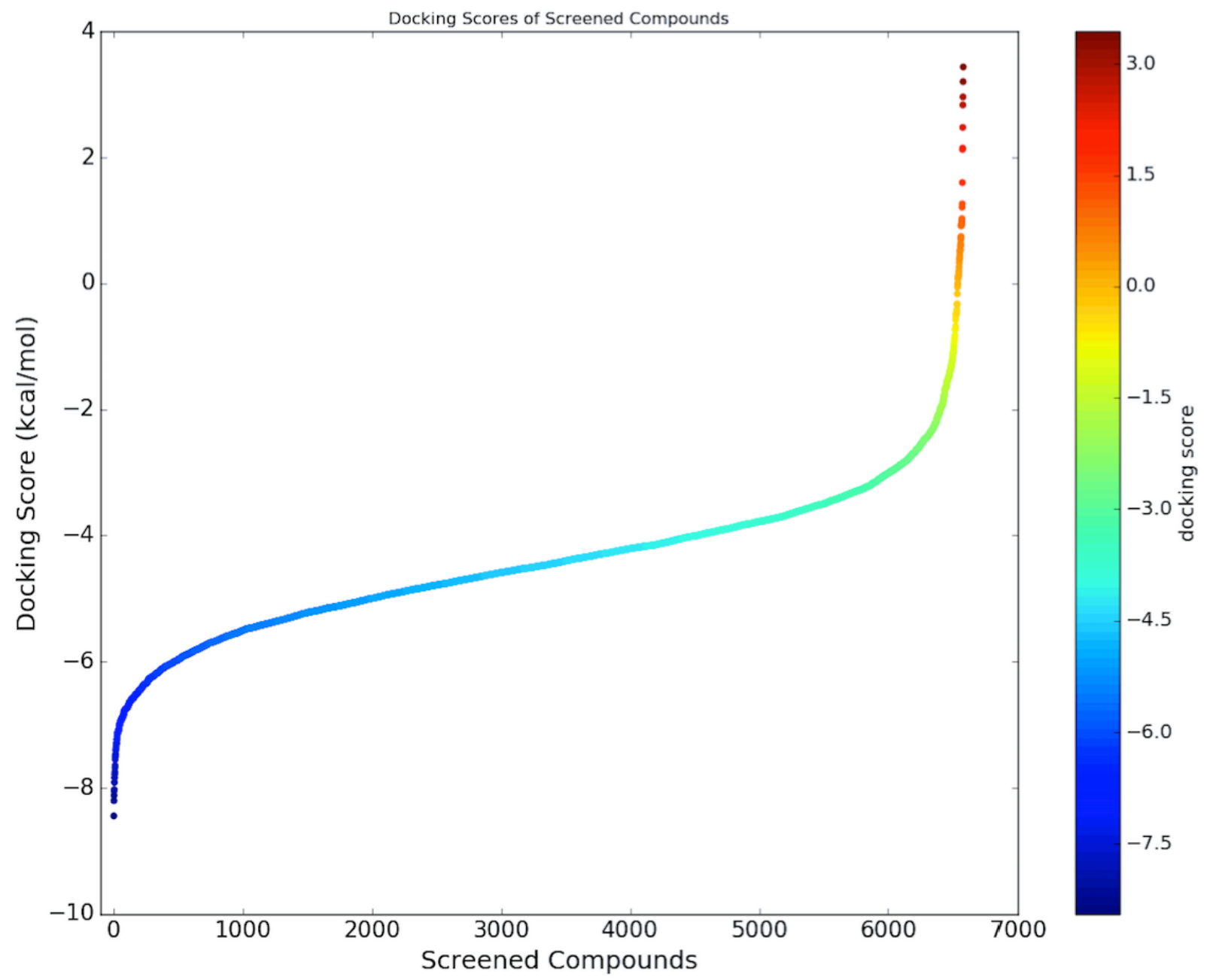

Figure S2. Docking scores of all screened compounds at the binding pocket of TMPRSS2. 


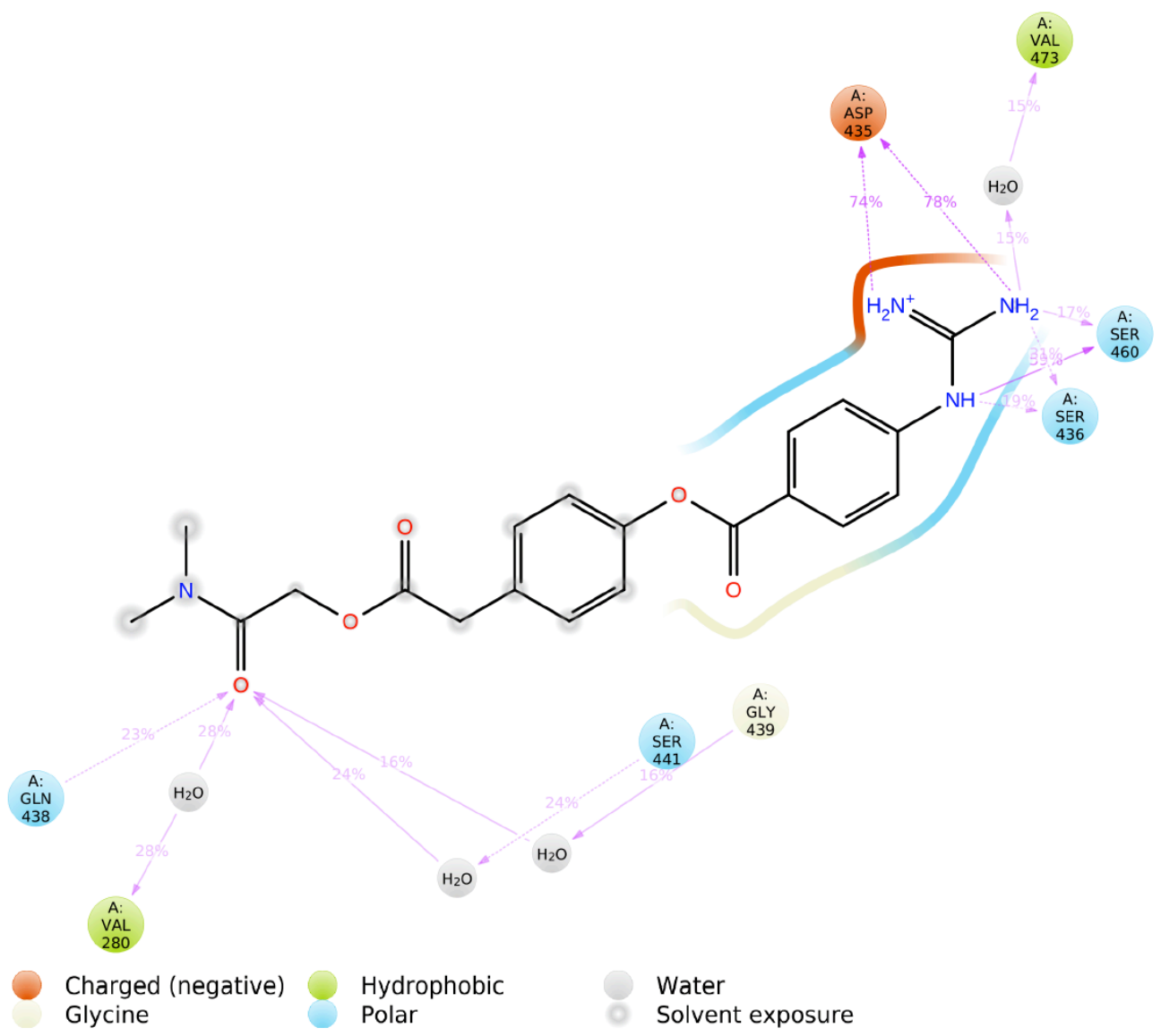

Figure S3. 2D ligand interactions diagram of Camostat at the binding pocket of TMPRSS2. 


\begin{tabular}{|c|c|c|}
\hline Compounds & $\begin{array}{l}\text { Docking } \\
\text { Score } \\
\text { (kcal/mol) }\end{array}$ & $\begin{array}{l}\text { Average } \\
\text { MM/GBSA } \\
\text { (kcal/mol) }\end{array}$ \\
\hline Reproterol & -8.445 & -47.283 \\
\hline Ociltide & -8.204 & -57.549 \\
\hline Theodrenaline & -8.120 & -26.242 \\
\hline Hexafluronium bromide & -8.045 & -67.775 \\
\hline Cliropamine & -8.028 & -38.601 \\
\hline Dab-452 & -7.912 & -60.818 \\
\hline Fenoterol & -7.839 & -45.418 \\
\hline Frakefamide & -7.785 & -42.928 \\
\hline mitoxantrone & -7.749 & -44.749 \\
\hline Adimolol & -7.677 & -58.356 \\
\hline Mesuprina & -7.649 & -45.323 \\
\hline Esorubicin & -7.549 & -42.773 \\
\hline Arbutamine & -7.515 & -56.584 \\
\hline Penimepicycline & -7.499 & -44.513 \\
\hline $\begin{array}{l}\text { (Alpha.s)-alpha-[alpha-(2,4-dimethyl-9h-pyrido(2,3- } \\
\text { b)indol-9-yl)-p-tolyl]-n-((alpha.r)- } \\
\text { alpha(hydroxymethyl)benzyl)cyclopentaneacetamide }\end{array}$ & -7.463 & -64.419 \\
\hline $\begin{array}{l}\text { 1,2,3-Dihydro-5-benzo(b)thienyl-2-(4- } \\
\text { phenylbutylamino)-1-propanol }\end{array}$ & -7.407 & -44.191 \\
\hline Quinidine & -7.380 & -48.078 \\
\hline Tilisolol & -7.346 & -24.419 \\
\hline Primozida & -7.341 & -66.157 \\
\hline Ad 810 & -7.340 & -37.037 \\
\hline Carbuterol & -7.313 & -18.889 \\
\hline Pronetalol & -7.293 & -24.264 \\
\hline Vanyldisulfamide & -7.290 & -61.900 \\
\hline Difebarbamate & -7.235 & -76.184 \\
\hline Piroxicillin & -7.230 & -57.173 \\
\hline Dapoxetine & -7.187 & -49.660 \\
\hline Etanterol & -7.179 & -12.707 \\
\hline Zinterol & -7.167 & -42.080 \\
\hline Amosulalol & -7.145 & -45.639 \\
\hline Fenprostalene & -7.136 & -57.394 \\
\hline Carazolol & -7.128 & -37.720 \\
\hline Benzquercin & -7.119 & -96.802 \\
\hline Naftopidil & -7.113 & -56.904 \\
\hline Denopamine & -7.107 & -56.310 \\
\hline Brefonalol & -7.107 & -44.698 \\
\hline Nardeterol & -7.106 & -49.928 \\
\hline
\end{tabular}




\begin{tabular}{|l|l|l|}
\hline $\begin{array}{l}\text { 3-(Cis-2,6-dimethylpiperidino)-n-(4 } \\
\text { methoxybenzoyl)sidnonimine }\end{array}$ & -7.095 & -52.087 \\
\hline Nordefrin & -7.083 & -28.408 \\
\hline Ametantrone & -7.079 & -49.325 \\
\hline Epicainide & -7.078 & -60.527 \\
\hline Carteolol & -7.077 & -29.853 \\
\hline Cinacalcet & -7.058 & -52.311 \\
\hline N-benzoyl-I-tyrosyl-paba & -7.034 & -71.183 \\
\hline Panamesine & -7.013 & -56.305 \\
\hline Neraminol & -7.005 & -45.562 \\
\hline Bucindolol & -7.003 & -60.390 \\
\hline Unii-f6s9cmy4se & -7.002 & -63.674 \\
\hline Giripladib & -6.975 & -64.455 \\
\hline Pancopride & -6.969 & -52.768 \\
\hline Nolinium bromide & -6.962 & -51.067 \\
\hline
\end{tabular}

Table S1. Docking scores of top-50 compounds at the TMPRSS2 binding site. These compounds were initially used in short (10-ns) MD simulations. Table also shows average MM/GBSA scores of these compounds from derived 1000-trajectories throughout the simulations. 